\title{
Robust Wavelength-Converting and Lasing Media from Wafer-Scale Inorganic Perovskites Enabled by a Protective Surface Layer
}

Yinjuan Ren, ${ }^{\dagger,}$ Weihua Wang, ${ }^{\dagger}$ Ziming Wang, ${ }^{+}$Siyang Xia, ${ }^{t,+}$ Yue Wang ${ }^{+*}$

${ }^{\dagger}$ MIIT Key Laboratory of Advanced Display Materials and Devices, Institute of

Optoelectronics \& Nanomaterials, College of Materials Science and Engineering, Nanjing University of Science and Technology, Nanjing 210094, China

tDepartment of Chemistry, National University of Singapore, 3 Science Drive 3, Singapore 117543, Singapore

* To whom correspondence should be addressed, email: ywang@njust.edu.cn 


\section{Methods}

Fabrication of $\mathrm{CsPbBr}_{3}$ wafer: The $\mathrm{CsPbBr}$. perovskite crystals were fabricated by reacting $\mathrm{CsBr}(42.6 \mathrm{~g})$ and $\mathrm{PbBr}_{2}(73.4 \mathrm{~g})$ in a fused silica ampoule. The $\mathrm{CsPbBr}$ polycrystalline was firstly synthesized for uniform reaction. After that, the ampoule was put into the Bridgman furnace with the aim to grow the perovskite ingot. The furnace temperature was set as $60{ }^{\circ} \mathrm{C}$ higher than the melting point of $\mathrm{CsPbBr}_{3}$. Therefore, the convex solid-liquid interface can be obtained. Finally, the $\mathrm{CsPbBr}$ ingot was cut into wafers with thickness of $\sim 1 \mathrm{~mm}$.

Optical characterization: Stimulated emission from $\mathrm{CsPbr}_{3}$ wafer was measured by a home-build optical system, where the excitation wavelength can be tuned from $260 \mathrm{~nm}$ to $2.6 \mu \mathrm{m}$. The pump fluence was adjusted by an attenuator. The emission signal was collected by an optical fiber and detected by a charge-coupled device (CCD) equipped with a monochromator. The emission dynamics was measured by a streak camera system. The temporal resolution of the system was $\sim 30 \mathrm{ps}$.

Fabrication of wavelength-converting and laser device: The device was built by sandwiching the $\mathrm{CsPbBr}_{3}$ bulk crystals between two parallel and highly reflective mirrors which served as the cavity resonator. The pump source at optical wavelength of $800 \mathrm{~nm}$ was directed on the device in the vertical direction. The pump fluence was adjusted by an attenuator. The emission signal was collected by an optical fiber and detected by a charge-coupled device (CCD) equipped with a monochromator. 


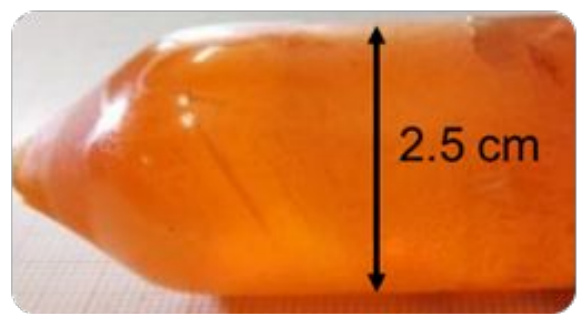

Figure S1. Photograph of the fabricated $\mathrm{CsPbr}_{3}$ perovskite crystals or ingot.

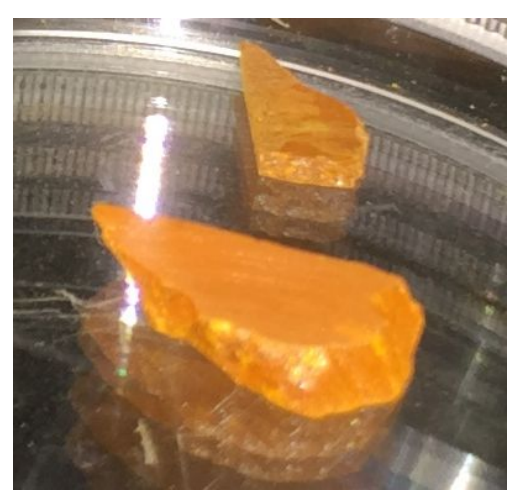

Figure S2. Photograph of the sub-centimeter-sized $\mathrm{CsPbBr}_{3}$ crystals obtained by breaking the $\mathrm{CsPbBr}_{3}$ wafer. 


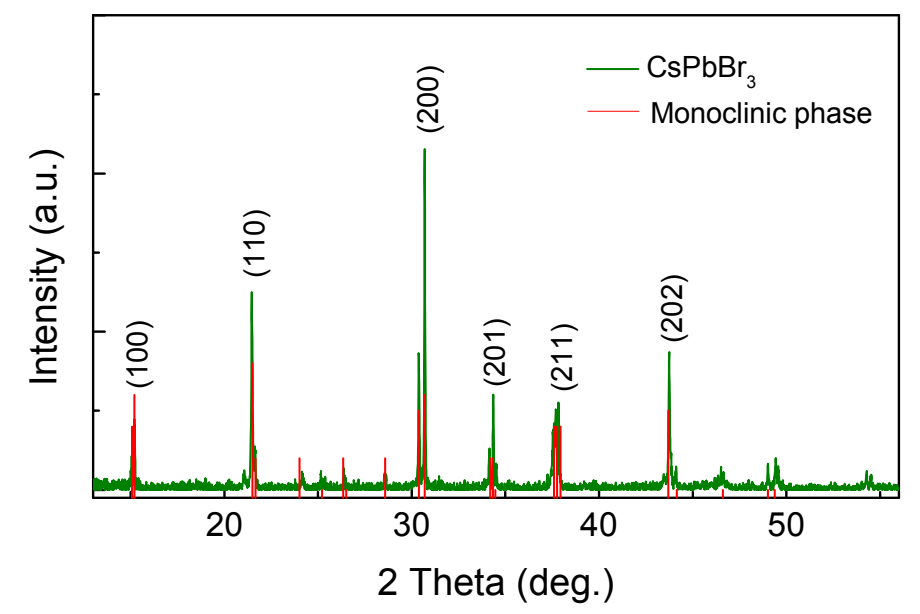

Figure S3. X-ray diffraction pattern of the fabricated $\mathrm{CsPbBr}_{3}$ crystal, exhibiting the monoclinic phase. 

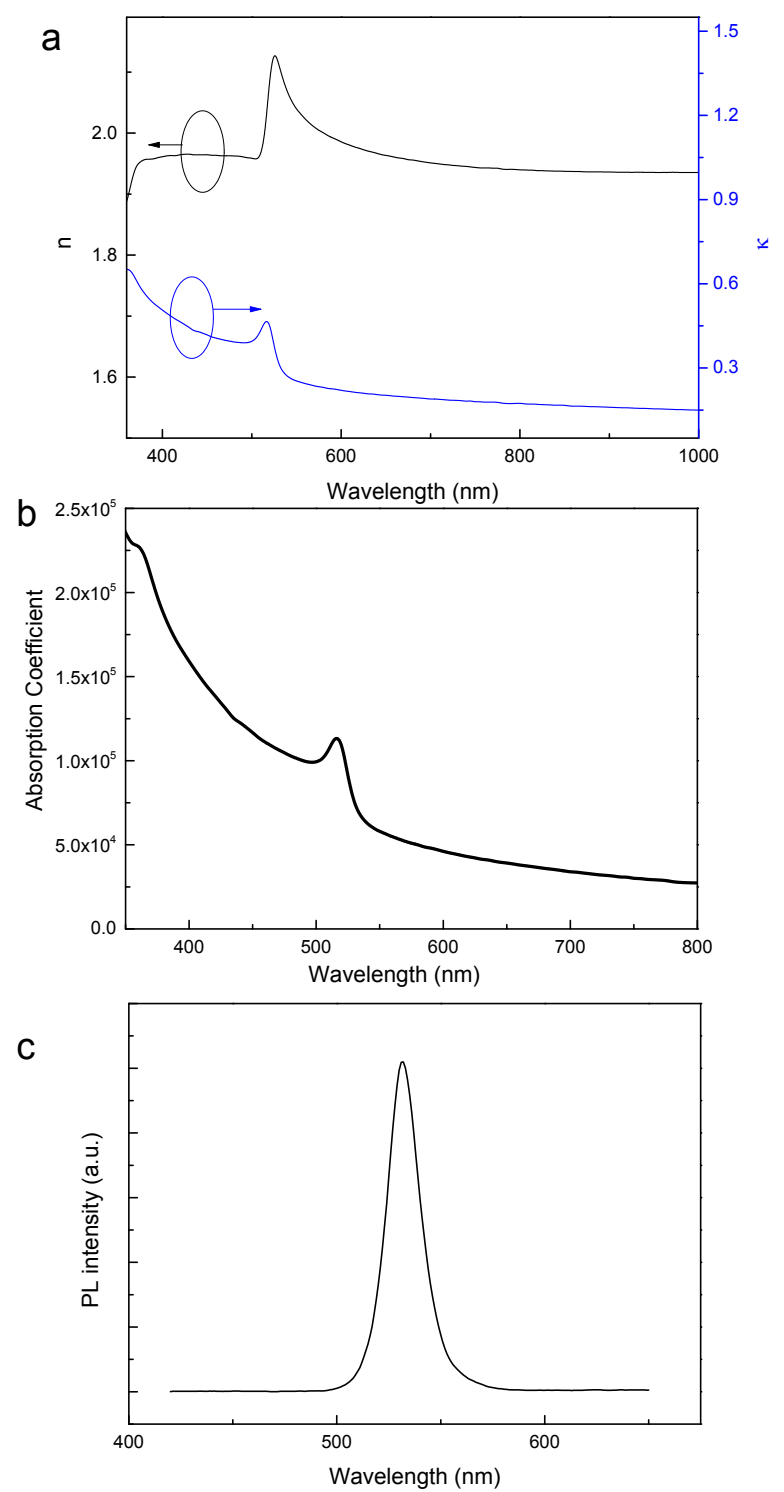

Figure S4. (a) Plot of $n-\kappa$ diagram of the fabricated $\mathrm{CsPbBr}_{3}$ crystal. (b) Absorption spectrum obtained from the $n-\kappa$ diagram of $\mathrm{CsPbBr}_{3}$ crystal. (c) PL spectrum of the fabricated $\mathrm{CsPbBr}_{3}$ crystal. 


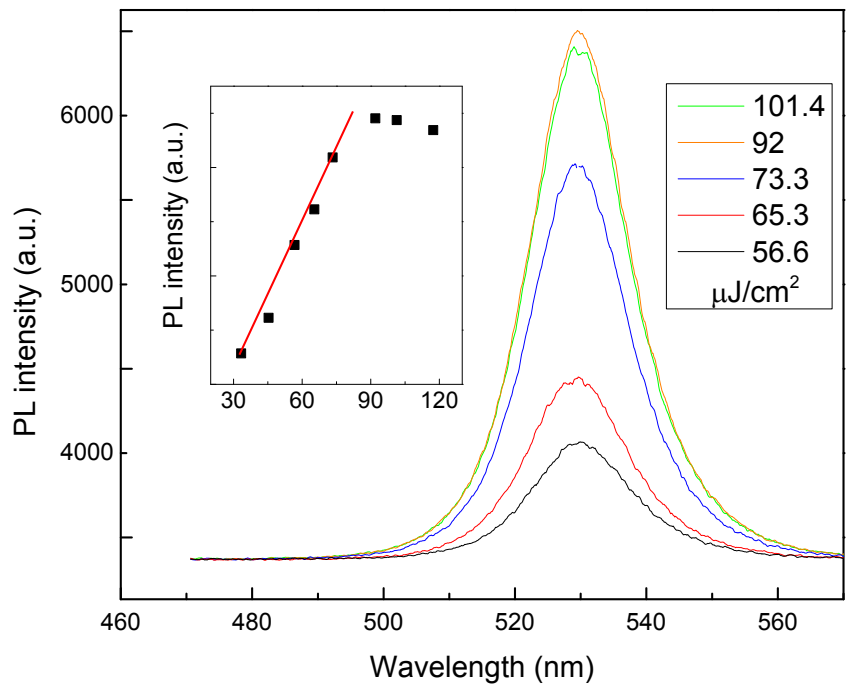

Figure S5. PL spectra from $\mathrm{CsPbBr}_{3}$ crystal under excitation wavelength of $400 \mathrm{~nm}$ and varied pump fluences.

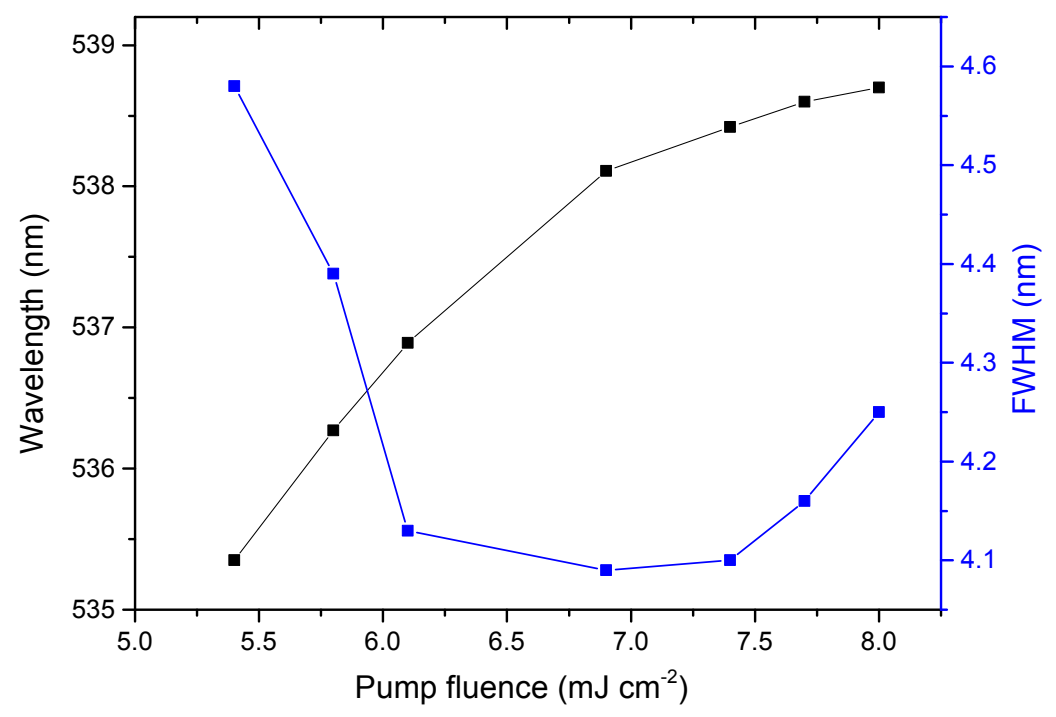

Figure S6. Variation of stimulated emission peak wavelength and linewidth as a function of pump fluence. 
Table S1. Summary of stimulated emission (SE) and lasing performance from inorganic lead halide perovskites (ILHPs) by two photon pumping.

\begin{tabular}{lccc}
\hline ILHPs & SE threshold & Lasing threshold & Stability \\
\hline Colloidal quantum dots ${ }^{1-3}$ & $12 \mathrm{~mJ} \mathrm{~cm}^{-2}$ & $0.9 \mathrm{~mJ} \mathrm{~cm}^{-2}$ & $<2$ hour \\
Nano/microwire/nanorods & & & \\
Microplates & & & \\
& & & \\
Perovskite wafer (this work) & $5.2 \mathrm{~mJ} \mathrm{~cm} \mathrm{~cm}^{-2}$ & $3.7 \mathrm{~mJ} \mathrm{~cm}^{-2}$ & $>4$ hours \\
\hline
\end{tabular}




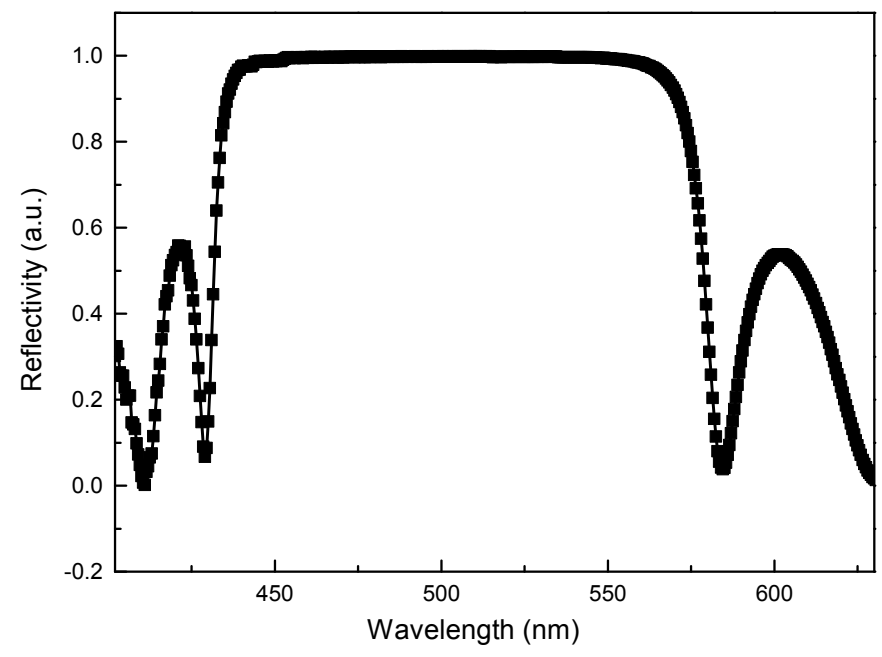

Figure S7. The wide-band reflection spectrum of the mirror used for the laser construction. 


\section{References}

1. Pan, J.; Sarmah, S. P.; Murali, B.; Dursun, I.; Peng, W.; Parida, M. R.; Liu, J.; Sinatra, L.; Alyami, N.; Zhao, C.; Alarousu, E.; Ng, T. K.; Ooi, B. S.; Bakr, O. M.; Mohammed, O. F., Air-Stable Surface-Passivated Perovskite Quantum Dots for Ultra-Robust, Single- and Two-Photon-Induced Amplified Spontaneous Emission. The Journal of Physical Chemistry Letters 2015, 6, 5027-5033.

2. Xu, Y.; Chen, Q.; Zhang, C.; Wang, R.; Wu, H.; Zhang, X.; Xing, G.; Yu, W. W.; Wang, X.; Zhang, Y.; Xiao, M., Two-Photon-Pumped Perovskite Semiconductor Nanocrystal Lasers. Journal of the American Chemical Society 2016, 138, 3761-3768.

3. Wang, Y.; Li, X.; Zhao, X.; Xiao, L.; Zeng, H.; Sun, H., Nonlinear Absorption and Low-Threshold Multiphoton Pumped Stimulated Emission from All-Inorganic Perovskite Nanocrystals. Nano Letters 2016, 16, 448-453.

4. Wang, X.; Zhou, H.; Yuan, S.; Zheng, W.; Jiang, Y.; Zhuang, X.; Liu, H.; Zhang, Q.; Zhu, X.; Wang, X.; Pan, A., Cesium lead halide perovskite triangular nanorods as high-gain medium and effective cavities for multiphoton-pumped lasing. Nano Research 2017, 10, 3385-3395.

5. He, H.; Ma, E.; Chen, X.; Yang, D.; Chen, B.; Qian, G., Single Crystal Perovskite Microplate for High-Order Multiphoton Excitation. Small Methods 2019, 3, 1900396. 